\title{
Online-Ressourcen
}

\section{ALIQUOT - Un atlante digitale interattivo online per lo studio e la visualizzazione delle varianti della lingua italiana di uso quotidiano, <www.atlante-aliquot.de>}

\section{Introduzione}

«Marinare la scuola, chi non l'ha mai fatto? Probabilmente si può affermare che la stragrande maggioranza degli studenti abbia fatto almeno una volta nella vita quest'esperienza. Eppure quasi nessuno utilizza il verbo marinare. Che espressioni usano gli italiani per dare un nome a quel sentimento di rubata libertà misto al pericolo di essere scoperti da genitori o insegnanti? Far berna, far sega, far vela, bigiare, bruciare, forcare, far forca, far filone, saltare...?».

Con queste parole si presenta all'utente il progetto ALIQUOT (<www.atlantealiquot.de>), il primo atlante digitale interattivo online per lo studio e la visualizzazione delle varianti della lingua italiana quotidiana.

L'italiano di uso quotidiano, fortemente correlato con il cosiddetto italiano regionale, è uno dei fenomeni che più caratterizzano la lingua italiana. Ciononostante, in tale ambito, si registra solamente una serie di studi a livello regionale o locale come quella riportata da Telmon a conclusione del suo volume Guida allo studio degli italiani regionali (cf. Telmon 1990, 199-277). Esiste inoltre qualche studio più recente, come ad esempio quello di Antonini/Moretti (2000) sull'italiano regionale nel Canton Ticino o quello di Cerruti (2007) nel campo delle perifrasi verbali. In ambito lessicale, stupisce (per quanto ci è dato sapere) l'assenza di un atlante che raffiguri la distribuzione dei geosinonimi dell'italiano. Un'eccezione è rappresentata dal Vocabolario della lingua italiana Treccani (2009) che, in appendice alla versione stampata come in quella online e in CD-ROM, riporta un elenco di circa un centinaio di geosinonimi visualizzati tramite carte geolinguistiche. Ciò che nelle carte del vocabolario della Treccani manca è però la georeferenziazione precisa delle diverse varianti lessicali all'interno del Paese, cosicché queste sono solo difficilmente utilizzabili per scopi scientifici di studio della variazione linguistica italiana. ${ }^{1}$

1 Teniamo a precisare in questa sede che non è assolutamente nostra intenzione criticare il lavoro della Treccani. Siamo chiaramente coscienti del fatto che il fine che la rinomata enciclopedia si è posta nella visualizzazione dei geosinonimi in Italia non è tanto quello di creare carte geografiche precise ad uso di linguisti quanto quello divulgativo. Tale lavoro, anzi, offre un validissimo spunto per nuove ricerche quale ad esempio ALIQUOT che, grazie alla Treccani, trova l'ispirazio- 
Tale «vuoto» di ricerca lascia particolarmente sbalorditi se si pensa che Trifone (2010, 158), elaborando i dati del Grande Dizionario Italiano dell'uso (GRADIT), arriva ad affermare che tra il 1951 e il 2000 nell'italiano di uso comune si sono diffuse 1664 parole dialettali o regionali.

Il progetto ALIQUOT (Castellarin/Tosques 2012), nato durante il convegno «20 anni di geolinguistica digitale» ${ }^{2}$ tenutosi presso la Humboldt-Universität zu Berlin il 2 e 3 novembre 2012 dall'idea di Stephan Elspaß e Robert Möller per il loro AdA (Atlas zur deutschen Alltagssprache), ${ }^{3}$ si propone di colmare questo vuoto di ricerca elaborando i dati raccolti al fine di una rappresentazione cartografica dei geosinonimi dell'italiano attraverso una precisa georeferenziazione delle risposte degli informanti.

\section{Cenni metodologici}

\subsection{La raccolta dati per inchiesta indiretta}

Per la raccolta dei dati, ALIQUOT utilizza la metodologia d'indagine indiretta di tipo quantitativo che permette un afflusso consistente di dati da tutto il territorio nazionale e che si è rivelata particolarmente proficua per ottenere un alto numero di informanti, altrimenti, a breve termine, irraggiungibile. Molti studiosi sono d'accordo nel sostenere i vantaggi di questo metodo di inchiesta. Uno per tutti è Eichhoff, che, a proposito della metodologia indiretta tramite posta scriveva:

«Die Entscheidung zugunsten der indirekten Methode wird bei Wortschatzuntersuchungen am leichtesten fallen. Hier kommen die Vorteile der schriftlichen Befragung [= indirekten Befragung] voll zur Geltung, während die Nachteile das Datenmaterial in seinen wesentlichen Aspekten nicht berühren» (1982, 550).

La nascita delle caselle di posta elettronica ha aumentato a dismisura i vantaggi, già menzionati da Eichhoff, di questo metodo, velocizzando moltissimo i tempi di inchiesta e abbattendo drasticamente i costi che l'affrancatura delle lettere portava con sé - già risibili se confrontati con i costi che si devono affrontare per inchieste dirette.

\footnotetext{
ne per i lessemi che compaiono nelle diverse inchieste e che si appoggia a questo lavoro per garantire la confrontabilità dei dati raccolti e controllarne così la consistenza.

$2<$ http://www2.hu-berlin.de/vivaldi/tagung/beitraege/pdf/inhaltsverzeichnis.html >.

$3<\mathrm{http}$ ///www.atlas-alltagssprache.de/>.
} 
ALIQUOT si serve di questionari compilabili dagli informanti direttamente online. Tale metodologia si dimostra, rispetto alle precedenti, ancora più rapida, sia dal punto di vista della raccolta dati, sia soprattutto per quanto riguarda il controllo e l'elaborazione di questi. Tramite l'inserimento diretto delle risposte fornite dagli informanti stessi in una banca dati computerizzata il controllo ex post delle stesse e la seguente creazione di carte geolinguistiche avvengono pressoché automaticamente.

La scelta di proporre agli informanti un questionario online si propone di sfruttare tutti i vantaggi della metodologia di inchiesta indiretta ed è basata sulla necessità di raccogliere dati da una superficie il più vasta possibile nel minor tempo possibile. Ci si ispira così (cf. Castellarin/Tosques 2012, 252) a Georg Wenker, secondo il quale è meglio raccogliere una piccola quantità di dati da tutto il territorio nazionale che una grande quantità di dati con un'inchiesta che presenta una rete di punti a maglie troppo larghe (cf. Mitzka 1938, 45). L'alta partecipazione da parte degli italiani alle inchieste di ALIQUOT, e il conseguente cospicuo numero di dati raccolti, ha contribuito a validare la scelta di tale metodo.

\subsection{La ricerca degli informanti}

La ricerca degli informanti si basa in primo luogo sulla «tecnica del passaparola». In un primo tempo sono stati contattati via e-mail tutti i nostri amici, conoscenti, parenti e colleghi italiani ai quali è stato chiesto di compilare il questionario e di diffonderlo a loro volta tra i loro contatti. Questo metodo di ricerca degli informanti si è rivelato tuttavia, nello specifico caso di ALIQUOT, in realtà un po' deludente.

Per ovviare al problema della lentezza nella ricerca degli informanti con il metodo del passaparola, si è creato un account in uno dei maggiori social network del mondo. Grazie all'utilizzo di Facebook, si è arrivati ad ottenere un numero di quasi 900 informanti nella prima inchiesta, di oltre 1400 per la seconda, poco più di 1000 per la terza e quasi 1700 per la quarta inchiesta. In un prossimo futuro si pensa di pubblicare qualche carta geolinguistica su giornali quotidiani o riviste di divulgazione scientifica in Italia, allo scopo di aumentare la popolarità dello studio nel Paese, guadagnando così ulteriori informanti che partecipino anche alle inchieste future. Infine, la partecipazione a diverse conferenze quali il Romanistentag (Würzburg 2013) o il FJR di Berna (marzo 2014) ${ }^{4}$ e l'Euralex (Bolzano

$4<$ http://www.kas.unibe.ch/fjr2014/pdf/reader.pdf>. 
2014), rappresenta un'occasione sia per sottoporre ALIQUOT alla comunità scientifica, sia per ottenere nuovi informanti. La ricerca degli informanti avviene così in modo «casuale»; non esiste né la possibilità di influire sul tipo di informante che risponde di volta in volta al questionario, né di controllare in precedenza se l'informante sia attendibile o meno, o se rispecchi alcune caratteristiche desiderate. Se questo può essere un limite dell'inchiesta indiretta, si è però anche dell'opinione che solo con un principio di casualità nella ricerca degli informanti sia possibile ottenere risposte che rispecchino la fattuale varietà linguistica nella Penisola che altrimenti, attraverso la ricerca di informanti ad hoc, potrebbe risultare, anche involontariamente, falsata dall'esploratore stesso.

\subsection{I questionari dei turni d'inchiesta}

Il progetto ALIQUOT propone ai suoi informanti due inchieste all'anno; ogni inchiesta si compone di un numero che varia tra le 10 e le 15 domande circa, più una breve parte finale nella quale viene chiesto agli informanti di inserire oltre al CAP della città per cui sono valide le risposte date, anche alcuni dati socio-demografici.

Tutte le domande di ALIQUOT sono formulate in maniera tale da evitare di suggerire all'informante la parola dell'italiano standard. Si è inoltre cercato di utilizzare formulazioni il più neutre possibile, volendo evitare le difficoltà connesse con la dizione italiano regionale e spostando fortemente l'accento sulla dimensione quotidiana della lingua in determinate zone d'Italia. Anche qui una precisazione è d'obbligo: non si fa riferimento alla regione e alla regionalità dei lessemi richiesti e si domanda agli informanti di rispondere solo per la propria città o paese. Si prenda ad esempio la domanda per il lessema lavorare. In ALIQUOT è così formulata:

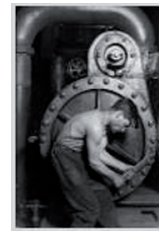

2.Quale espressione si sente normalmente nella tua città o nel tuo paese per esercitare un impiego o una professione?

Fig. 1: Esempio di domanda dal questionario ALIQUOT (prima inchiesta): <http://www.atlante-aliquot.de/primo_turno.php〉.

La formulazione della domanda pone inoltre un forte accento sulla dimensione del parlato. Con questa scelta ci si rifà a Cortelazzo $(2001,30)$ il quale ritiene che l'italiano regionale sia «un fenomeno più afferente all'oralità che alla scrittura». 
Ogni domanda di ALIQUOT è correlata da una foto ${ }^{5}$ che la illustra o la chiarisce. La decisione di utilizzare questa forma di presentazione ha un duplice scopo; in primo luogo la foto serve per disambiguare quelle domande che alla lettura potrebbero risultare troppo complicate o ambigue, o d'altra parte, come puro riferimento per quelle domande per le quali non si è voluto dare una definizione che sarebbe risultata davvero troppo lunga o complessa (ad esempio per il lessema lucertola della quinta inchiesta la domanda di ALIQUOT è: «Quale espressione si sente normalmente nella tua città o nel tuo paese per l'animale raffigurato nella foto?»). In secondo luogo, la presentazione multimediale influisce positivamente sulla motivazione degli informanti che percepiscono il questionario come più breve. La brevità agisce in modo positivo sulla concentrazione e conseguentemente contribuisce alla diminuzione del numero di errori commettibili (cf. Atteslander 2004).

Le domande del questionario di ALIQUOT sono a risposta multipla. La rosa delle possibili risposte offerte ha lo scopo di orientare l'informante nella scelta, in modo da non ottenere solo risposte riconducibili al dialetto locale o solo all'italiano standard. Ciononostante, tra le possibili risposte compaiono sempre la denominazione dell'italiano standard così come denominazioni di stampo più dialettale; si evitano però quelle espressioni che, per le loro caratteristiche morfologiche, appartengono chiaramente al dialetto. In ogni item è stato aggiunto alle possibili risposte un campo denominato, come di consueto, altro, in cui gli informanti possono inserire un termine non presente nella lista, nel caso che nessuna delle proposte sia soddisfacente.

Nella seconda parte dell'inchiesta, dopo aver risposto alle domande, gli informanti sono pregati di inserire alcuni dati socio-demografici (luogo per cui si risponde, età, titolo di studio, lingua parlata in famiglia e con gli amici, etc.) di particolare interesse per il progetto.

Poiché lo scopo principale di ALIQUOT risiede nella creazione di un atlante della variazione linguistica della lingua italiana quotidiana, di fondamentale importanza scientifica è la georeferenziazione esatta dei punti d'inchiesta. Tramite il CAP, il nome della città/paese, e della nazione otteniamo, con l'aiuto di Google Maps, la longitudine e la latitudine del punto geografico preciso per cui ogni informante ha risposto.

Per questo motivo, chiunque abbia risposto alle domande, deve inserire nella seconda parte del questionario il nome del luogo per cui sono valide le risposte date ed il relativo CAP.

5 Tutte le immagini presenti nei questionari di ALIQUOT sono prese da Wikipedia o sono foto scattate o disegni creati dai responsabili del progetto. Questo accorgimento evita di incorrere in problemi risultanti dall'utilizzo di immagini protette da copyright e quindi non liberamente utilizzabili. Vedi ad esempio: <www.atlante-aliquot.de/quinto_turno.php>. 
Chiudono il questionario due campi a riempimento facoltativo, in cui si chiede agli informanti di specificare una o più espressioni che desidererebbero venisse/ro studiata/e e di esternare qualche commento o critica sul progetto. Questi due campi sono stati inseriti principalmente a scopo motivazionale e per «fidelizzare» gli informanti. Nella preparazione delle inchieste successive, poi, si tiene conto dei lessemi proposti dagli informanti stessi e si tenta di inserirne il maggior numero possibile compatibilmente con i criteri di comparabilità con altre ricerche precedenti, gli interessi particolari di ALIQUOT e la «proponibilità» della domanda per un vasto pubblico che può comprendere anche minorenni. ${ }^{6}$

\subsection{La pubblicazione dei dati}

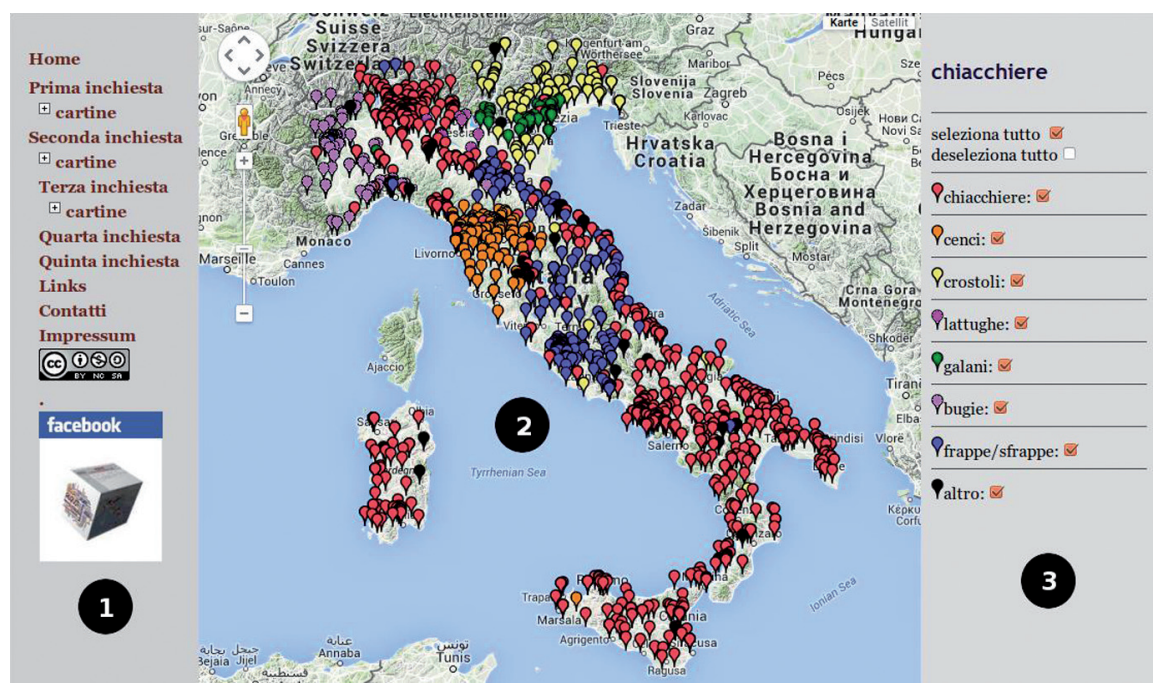

Fig. 2: ALIQUOT - Esempio di carta geolinguistica (quarta inchiesta, dolce di carnevale).

\footnotetext{
6 Alcune richieste sono risultate, almeno per il momento, improponibili. Un informante, ad esempio, ha espresso il suo interesse per i geosinonimi di eiaculare. L'inserimento di questo item, volendo correlare di una foto ogni lessema richiesto, risulta particolarmente arduo. Non si vuole infatti ferire la sensibilità e il senso del pudore di nessuno. Nonostante questa espressione sia senza dubbio interessante e ci sia da aspettarsi un'ampia variazione all'interno del Paese, si è deciso di non inserirla nelle seguenti inchieste. Forse in futuro si arriverà ad una soluzione accettabile del dilemma.
} 
Sul sito internet del progetto ALIQUOT ${ }^{7}$ alle voci «prima inchiesta (cartine)», «seconda inchiesta (cartine)» e «terza inchiesta (cartine)» (fig. 2: (1) è possibile visualizzare le carte geolinguistiche pubblicate online. Per la presentazione e la pubblicazione dei dati, il progetto ALIQUOT si serve del supporto di Google Maps. Cliccando sulle domande si aprono carte geolinguistiche interattive (fig. 2: (2), in cui ad ogni geosinonimo viene attribuito un colore, la cui scelta non è casuale. Nelle carte - dove questa operazione risulta possibile - si sono utilizzati colori caldi per quei geosinonimi che più si avvicinano all'italiano standard e colori via via più freddi per quelli che gradualmente si allontanano dalla varietà standard della lingua e assumono sfumature più dialettali. Il colore nero è riservato a quei geosinonimi contrassegnati nelle carte dalla voce altro. In questi casi si tratta di risposte di informanti che non sono uniformate con le risposte circostanti e che rappresentano così casi unici.

Data la complessità di alcune carte, che presentano (come nel caso dei sinonimi di marinare la scuola) più di venti espressioni diverse distribuite in tutta la Penisola, la gradazione del colore rispetto alla vicinanza del geosinonimo all'italiano standard risulta impossibile; qui i colori sono assegnati casualmente.

Sulla sinistra della carta (fig. 2: (1) compare il menu principale del sito, sulla destra invece (fig. 2: 3) sono visibili il titolo della carta e la lista dei geosinonimi relativi ad un determinato lessema.

Le risposte dei partecipanti sono raggruppate per lessema. Tale raggruppamento implica una certa riduzione della varietà di risposte forniteci dagli informanti. Si è scelto comunque di operare questa riduzione perché in alcuni casi gli informanti di una zona usano lo stesso termine per definire un determinato oggetto, ma gli uni ne utilizzano una forma più marcatamente dialettale degli altri. ${ }^{8}$ Lo scopo principale di ALIQUOT non è quello di documentare la variazione dialettale all'interno del Paese, già cartografata in due capolavori quali l'AIS e l'ALI e attualmente dal progetto VIVALDI, ${ }^{9}$ ma quello di rappresentare le variazioni della lingua di uso quotidiano, ancora assenti nel panorama degli atlanti linguistici nazionali. In ogni caso, non ci è sembrato opportuno escludere com-

$7<$ www.atlante-aliquot.de>.

8 È questo ad esempio il caso del lessema topo in Sardegna. Alcuni informanti hanno risposto con madrona, altri con madroà e altri ancora con merdona. Tutti questi lessemi sono stati raggruppati nella carta sotto la voce merdona presente anche nel Dizionario etimologico sardo (cf. Wagner 1960). Un altro caso simile riguarda il lessema anguria. In Calabria compaiono i lessemi zuparico, zipangolo e zipangulu. Anche qui le tre varianti sono state rappresentate con un medesimo colore. Tale operazione si è resa necessaria per garantire una certa leggibilità delle carte di ALIQUOT.

$9<$ http://www2.hu-berlin.de/vivaldi/?id=0001\&lang=it >. 
pletamente anche un'analisi del primo tipo. È stata pertanto inserita la possibilità di controllare, per ogni punto della rete d'inchiesta, la risposta precisa dell'informante. Cliccando su una delle puntine colorate che marcano i luoghi per cui è stato risposto si apre una finestrella in cui compaiono il nome del luogo, la risposta precisa, l'età e il sesso dell'informante.

L'interattività delle carte, ossia la possibilità da parte del fruitore di visualizzare solo i geosinonimi desiderati lasciando scomparire gli altri (fig. 2: (3), è stata inserita come risposta ad un problema pratico; in carte dove il numero di geosinonimi utilizzato è particolarmente alto, è pressoché impossibile distinguere a prima vista le zone in cui una determinata espressione compare. La possibilità di attivare nelle carte uno o più geosinonimi singolarmente, nascondendo gli altri, facilita molto non solo l'utilizzo e la comprensione di queste, ma anche il lavoro del ricercatore nell'analisi del materiale raccolto.

Succede così che spesso per uno stesso punto d'inchiesta si sovrappongano le risposte di più informanti. Il numero di CAP presenti nelle città italiane, infatti, non è così alto come ad esempio in quelle tedesche e quindi per uno stesso CAP sono rappresentate zone abbastanza ampie. In questi casi il colore che compare sulla carta generale è quello dato dalla risposta di maggioranza tra quelle degli informanti che hanno risposto per quel CAP. L'interattività delle carte può portare però alla luce le risposte differenti nascoste nella carta generale.

La scelta di pubblicare i dati in carte interattive risponde altresì ad un'altra esigenza pratica. Come già menzionato, per ogni item gli informanti di ALIQUOT hanno la possibilità di marcare nel questionario una o più risposte. Ai fini della leggibilità delle carte risultanti solo raramente le «doppie risposte» vengono evidenziate con un colore ; $^{10}$ nella maggioranza dei casi la tonalità che compare è quella della risposta più affine alla maggioranza delle risposte degli informanti per la stessa zona e per le zone limitrofe. La possibilità, però, di controllare in ogni momento le risposte date tramite un semplice «clic» permette di scoprire quali siano i punti in cui sono presenti più lessemi.

Google Maps, oltre ai vantaggi già menzionati dei software open source, offre un'ulteriore funzione che per ALIQUOT risulta molto interessante: la possibilità di zoomare rende possibile la rappresentazione della situazione linguistica in determinate regioni, province o addirittura città, visualizzando da vicino le differenze, a volte anche consistenti, tra quartiere e quartiere all'interno dello stesso centro urbano.

10 Questo avviene solo se in alcune macro-aree sono presenti per la maggioranza degli informanti due o più possibilità per uno stesso lessema. In questi casi la doppia scelta potrebbe essere effettivamente l'indice della presenza di una zona di transizione tra un regionalismo e l'altro o dell'espansione di un regionalismo a scapito della variante più tipica per la zona in questione. 


\section{Prospettive future}

Per concludere, uno sguardo al futuro. Il progetto ALIQUOT si limita (ora che è ancora nella sua fase iniziale) alla raccolta dati e alla loro pubblicazione online in forma di carte geolinguistiche messe a disposizione di chiunque abbia una connessione internet. Si rinuncia per ora ad uno studio più approfondito dei risultati ottenuti a causa di altri obblighi istituzionali e lavorativi. ${ }^{11}$

Non escludiamo assolutamente però la possibilità di dedicarci in un prossimo futuro ad un'analisi più approfondita dei nostri dati. Già oggi è possibile, scomponendo i dati raccolti, creare carte linguistiche differenziate in base ai dati sociodemografici dei nostri informanti. Sarà così possibile analizzare la lingua italiana quotidiana da un punto di vista sociolinguistico, campo che, per quanto ci è dato sapere, rimane ancora per gran parte inesplorato.

Un «cantiere» del progetto ALIQUOT a cui in effetti stiamo attualmente lavorando è il confronto diretto delle nostre carte con i dati raccolti dall'atlante dialettale AIS. Per rendere possibile tale confronto stiamo elaborando digitalmente le carte dell'AIS per rendere visibili attraverso Google Maps i confini geografici di determinati lessemi all'interno della Penisola. Sovrapponendo poi alla carta così creata la carta di ALIQUOT per il medesimo lessema sarà possibile visualizzare online l'espansione di un geosinonimo rispetto al lessema dialettale corrispondente (figure 3,4 e 5).

L'accennata distribuzione dei colori nelle carte in base alla vicinanza o meno di un determinato geosinonimo alla forma standard rappresenta inoltre un primo piccolo passo verso una futura dialettometrizzazione dei dati di ALIQUOT che permetterà una panoramica nazionale sulla situazione della lingua italiana quotidiana. Nel momento in cui si scrivono queste righe, tale operazione non è tuttavia ancora attuabile a causa del relativamente modesto numero di carte a disposizione.

Terminiamo con la speranza che ALIQUOT si possa rivelare un utile strumento per la ricerca linguistica e che sia così fonte di ispirazione e di dati per futuri studi sulla lingua italiana di uso quotidano.

11 Sia qui solo brevemente accennato il fatto che ALIQUOT è un progetto che non viene in alcun modo finanziato e che vive della passione dei suoi iniziatori per la lingua italiana quotidiana e dell'interesse dei molti informanti che costantemente partecipano alle inchieste a cui vorremmo in questa sede esprimere la nosta gratitudine! 

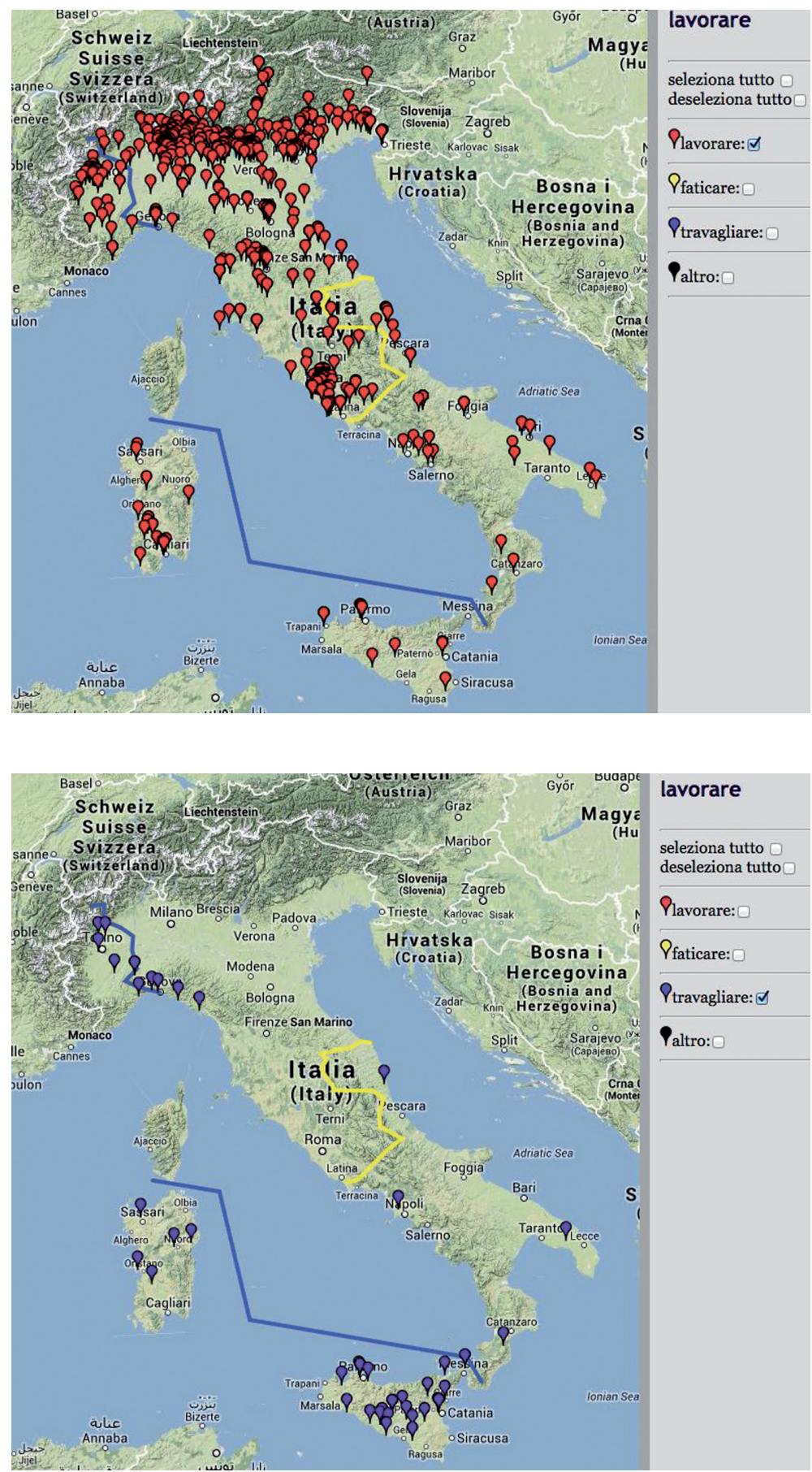


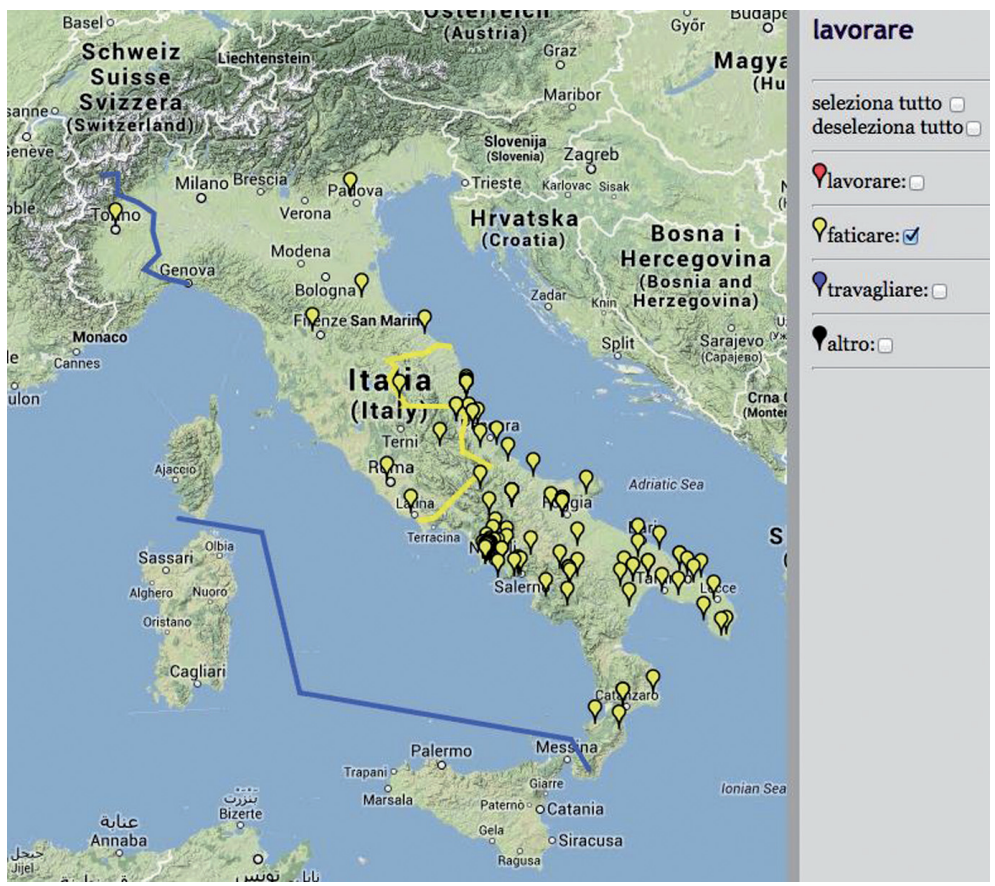

Fig. 3, 4 e 5: Sovrapposizione della carta ALIQUOT e AIS per il lessema lavorare.

\section{Bibliografia}

AdA = Elspaß, Stephan/Möller, Robert, Atlas zur deutschen Alltagssprache, 2001ss., <http://www.atlas-alltagssprache.de〉.

AIS = Jaberg, Karl/Jud, Jakob, Sprach- und Sachatlas Italiens und der Südschweiz, 8 voll., Zofingen, Ringier, Zofingen, 1928-1940.

$\mathrm{ALI}=$ Atlante Linguistico Italiano, Opera promossa dalla Società Filologica Friulana «G. I. Ascoli», diretta da Matteo G. Bartoli et al., Roma, Istituto Poligrafico e Zecca dello Stato. Libreria dello Stato, 1995ss. (<http://www.atlantelinguistico.it〉).

Antonini Francesca/Moretti Bruno, Le immagini dell'italiano regionale. La variazione linguistica nelle valutazioni dei giovani ticinesi, Locarno, Dadò, 2000.

Atteslander, Peter, Schriftliche Befragungen / Written Investigations, in: Ammon, Ulrich, et al. (edd.), Sociolinguistics / Soziolinguistik. An International Handbook of the Science of Language and Society / Ein internationales Handbuch zur Wissenschaft von Sprache und Gesellschaft, Berlin/New York, De Gruyter, 2004, 1063-1076.

Castellarin, Michele/Tosques, Fabio, ALIQUOT - L'Atlante della Lingua Italiana QUOTidiana, Rivista Italiana di Dialettologia. Lingue dialetti società 36 (2012), 245-262.

Castellarin, Michele/Tosques, Fabio, ALIQUOT - Atlante della Lingua Italiana QUOTidiana, in: Abel, Andrea/Vettori, Chiara/Ralli, Natascia (edd.), Proceedings of the XVI EURALEX Interna- 
tional Congress: The User in Focus, 15-19 July 2014, Bolzano / Bozen, 2014, 305-318, <http://euralex2014.eurac.edu/en/callforpapers/Pages/default.aspx〉.

Castellarin, Michele/Tosques, Fabio, Datenerhebung und elektronisches Publizieren geolinguistischer Daten in Theorie und Praxis, in: Gil, Alberto/Polzin-Haumann, Claudia (edd.), Kommunikations-und Diskussionsformen im 21. Jahrhundert. Beiträge zum Romanistentag 2013 in Würzburg, in corso di stampa.

Cerruti, Massimo, Sulla caratterizzazione aspettuale e la variabilità sociale d'uso di alcune perifrasi verbali diatopicamente marcate, Archivio Glottologico Italiano 92 (2007), 203-247.

Cortelazzo, Manlio, Riflessioni sull'italiano regionale, in: Fusco, Fabiana/Marcato, Carla (edd.), L'italiano e le regioni. Atti del Convegno di Studi di Udine, 15-16 giugno 2001, Udine, Centro Internazionale sul Plurilinguismo, Univ. degli Studi di Udine, 2001, 29-31.

Eichhoff, Jürgen, Erhebung von Sprachdaten durch schriftliche Befragung, in: Besch, Werner, et al. (edd.): Dialektologie. Ein Handbuch zur deutschen und allgemeinen Dialektforschung, 1. Halbband, Berlin/New York, De Gruyter, 1982, 549-553.

GRADIT = De Mauro, Tullio (ed.), Grande Dizionario Italiano dell'uso, 8 voll., Torino, UTET, 19992007.

Mitzka, Walther, Der deutsche Wortatlas, Zeitschrift für Mundartforschung 14 (1938), 40-55. Telmon, Tullio, Guida allo studio degli italiani regionali, Alessandria, Edizioni dell'Orso, 1990. Tosques, Fabio (ed.), 20 Jahre digitale Sprachgeographie. Tagungsband zur gleichnamigen Tagung, 2. bis 3. November 2012 in Berlin, Berlin, 2014, <http://www2.hu-berlin.de/vivaldi/ tagung/beitraege/pdf/inhaltsverzeichnis.html>.

Treccani, Vocabolario della lingua italiana, Roma, Istituto della Enciclopedia italiana fondata da Giovanni Treccani, Roma, 2009.

Trifone, Pietro, Storia linguistica dell'Italia disunita, Bologna, il Mulino, 2010.

VIVALDI = Kattenbusch, Dieter (ed.), Vivaio Acustico delle Lingue e dei Dialetti d'Italia (VIVALDI), 1999ss., <http://www2.hu-berlin.de/vivaldi>.

Wagner, Max L., Dizionario etimologico sardo, vol. 1: A-Y, Heidelberg, Winter, 1960.

Michele Castellarin: Humboldt-Universität zu Berlin, Institut für Romanistik, Unter den Linden 6, 10099 Berlin, E-Mail: michele_castellarin@yahoo.it Fabio Tosques: Humboldt-Universität zu Berlin, Institut für Romanistik, Unter den Linden 6, 10099 Berlin, E-Mail: ftosques@gmail.com 